\title{
Research Article \\ Clinical Significance and Prognostic Value of miR-28-5p in Colon Cancer
}

\author{
Ji-lin Li, ${ }^{1}$ Ke-zhi Li, ${ }^{1}$ Ming-zhi Xie, ${ }^{2}$ Yan-ping Tang, ${ }^{1}$ Yin-lin Tang, ${ }^{3}$ and Bangli $\mathrm{Hu} \mathbb{D}^{1}$ \\ ${ }^{1}$ Department of Research, Guangxi Medical University Cancer Hospital, Nanning 530021, China \\ ${ }^{2}$ Department of Chemotherapy, Guangxi Medical University Cancer Hospital, Nanning 530021, China \\ ${ }^{3}$ Clinical Laboratory, Maternal and Child Health Hospital of Guangxi, 530003 Nanning, Guangxi, China
}

Correspondence should be addressed to Bangli Hu; hubangli@gxmu.edu.cn

Received 17 January 2020; Accepted 28 April 2020; Published 20 May 2020

Academic Editor: Małgorzata Knaś

Copyright (c) 2020 Ji-lin Li et al. This is an open access article distributed under the Creative Commons Attribution License, which permits unrestricted use, distribution, and reproduction in any medium, provided the original work is properly cited.

Background. The association of miR-28-5p with colon cancer remains to be elucidated. This study aimed to determine the clinical significance and prognostic value of miR-28-5p in colon cancer. Methods. We retrospectively analyzed the data of miR-28-5p in colon adenocarcinoma data from The Cancer Genome Atlas (TCGA) and Gene Expression Omnibus (GEO), and the data was divided into cancer group and normal group, respectively. Forty colon cancer tissues and adjacent normal tissues were collected and tested by qRT-PCR methods. The difference of the miR-28-5p expression between colon cancer and normal tissues was compared. The clinical significance of miR-28-5p in colon cancer and the association with the survival were determined. The predictive value of miR-28-5p in clinical features was determined using receiver operating characteristic curve. The target genes of miR-28-5p were identified, and the functional of target genes was performed using bioinformatics analysis. Results: The expression of miR-28-5p was increased in colon cancer tissues compared with normal controls $(p=0.037)$. The expression of miR-28-5p was significantly increased in tissues with distant metastases compared with that without distant metastases $(p=0.026)$. Patients with high expression of miR-28-5p have a shorter survival time than those with low expression $(p=0.004)$. Cox analysis showed that miR-28-5p was an independent predictor for the survival of patients $(p=0.014)$. Combination of miR28-5p with TNM stage and clinical stage can improve the prognostic value for the patients $(p<0.05)$. miR-28-5p has a moderate predictive value in predicting the TNM stage and clinical stage ( $\mathrm{T}$ stage: $\mathrm{AUC}=0.515 ; \mathrm{N}$ stage: AUC $=0.523, \mathrm{M}$ stage: $\mathrm{AUC}=$ 0.572; clinical stage: AUC $=0.539)$. 711 potential target genes of miR-28-5p were screened; their function and pathways were identified. Conclusions: This study demonstrated that miR-28-5p was increased in colon cancer and can be an independent indicator for the overall survival in patients with colon cancer.

\section{Introduction}

Colorectal cancer (CRC) is one of the leading malignant cancers in the world, ranking third for incidence (10.2\%, with 1.8 million new cases) and second for mortality (9.2\%, with 881,000 deaths) of all cancers $[1,2]$. Colon cancer is a common subtype of CRC, and the survival of patients with colon cancer is varied greatly between early and advanced stage [3]. Thus, early detection and prediction the prognosis of colon cancer is crucial for the treatment in patients with colon cancer. Previous studies have reported that some biomarkers, including genes, miRNAs, were capable of early detecting and predicting the prognosis in patients with colon cancer
$[4,5]$. However, these results were often based on single center with few patients, which undermine the robustness of the results. Therefore, finding reliable biomarkers that could be used to early detect and predict the prognosis of colon cancer remains an urgent need for the doctors.

miRNAs are noncoding RNA molecules of 21-24 nucleotides that regulate the expression of target genes in a posttranscriptional manner. They have been implicated in many cancers and associated with clinical features, such as disease stage and survival in patients [6]. To date, some miRNAs were found to be valuable indicators for the prognosis for colon cancer. For example, miR-506 could greatly differentiate early-stage CRC from healthy individuals with $76.8 \%$ 
specificity and $60.7 \%$ sensitivity [7]. High miR-34a levels in CRC predict a rather increased risk for disease recurrence and poor overall survival, particularly in patients at an early TNM stage [8].

miR-28-5p has been implicated in several cancers, such as ovarian cancer [9], prostate cancer [10], and hepatocellular carcinoma [11]. Expression of miR-28-5p also found to be decreased in liver metastases tissues compared with the primary colorectal cancer tissues [12, 13]. More recently, Tsiakanikas et al. [14] report that miR-28-5p was downregulated in CRC compared with their adjacent noncancerous mucosae, suggesting that miR-28-5p was involving in the development of CRC. However, the current knowledge of miR-28-5p in colon cancer is limited. Thus, this study aimed to determine the clinical significance and potential prognostic value of miR-28-5p in colon cancer by using the data from The Cancer Genome Atlas (TCGA) and Gene Expression Omnibus (GEO) dataset and our colon cancer tissues.

\section{Materials and Methods}

2.1. Expression of $m i R-28-5 p$ in Colon Cancer from TCGA and GEO Database. In order to determine the association between miRNAs and the colon cancer, the data of colon adenocarcinoma (COAD) was downloaded from the TCGA database (https://cancergenome.nih.gov/). We also downloaded a colon cancer dataset (GSE49246) [15] from the GEO database. The COAD dataset from TCGA includes miRNA expression data from 453 colon cancer tissues and eight normal colon tissues. The GSE49246 dataset includes 40 colon cancer tissues and 40 adjacent normal tissues. The expression of miR-28-5p among gastrointestinal cancers, including COAD, esophageal cancer (ESCA), liver hepatocellular carcinoma (LIHC), rectal adenocarcinoma (READ), cholangiocarcinoma (CHOL), gastric cancer (STAD), was analyzed using OncomiR website online tool (http://www .oncomir.org), which uses the data from TCGA database.

2.2. Tissue Samples Collection. Forty colon cancer tissues and corresponding adjacent normal tissues were collected from the Biobank of Guangxi Medical University Cancer Hospital (Nanning, China) between January 2015 and December 2017. Fresh tissue samples were frozen within $30 \mathrm{~min}$ after surgery and stored in liquid nitrogen until use. The inclusion criteria were that the tissue histologically proven colon cancer, no severe major organ dysfunction, no prior cancer chemotherapy. This study was approved by the Ethics Committee of Guangxi Medical University Cancer Hospital; written informed consent was obtained from each patient.

2.3. RNA Isolation from Colon Cancer Tissues. Total RNA from colon cancer and adjacent normal tissues was isolated using TRIzol reagent (Invitrogen; Thermo Fisher Scientific, Inc., Waltham, MA, USA) according to the manufacturer's protocol. RNA concentration was measured using NanoDrop ND-1000 (Thermo Fisher Scientific, Inc.), and the quality was assessed using electrophoresis with $1.5 \%$ dena-
TABLE 1: Characteristic of the patients with colon cancer in this study.

\begin{tabular}{lccc}
\hline & TCGA data & GEO data & Our date \\
\hline $\begin{array}{l}\text { Age } \\
\text { Gender }\end{array}$ & $69(31-98)$ & $65(32-83)$ & $57(31-84)$ \\
$\quad$ Male/female & $269 / 244$ & $21 / 19$ & $22 / 18$ \\
$\begin{array}{l}\text { Differentiation } \\
\quad \text { Low/moderate/high }\end{array}$ & & \\
Location & & $8 / 28 / 4$ \\
$\quad$ Right/left site & $284 / 229$ & $18 / 22$ \\
$\begin{array}{l}\text { T stage } \\
\quad \text { T1/T2/T3/T4 }\end{array}$ & $9 / 85 / 355 / 63$ & & \\
N stage & & \\
$\quad$ N0/N1/N2 & $298 / 121 / 84$ & & \\
M stage & & & \\
$\quad$ M0/M1/MX & $381 / 77 / 55$ & & \\
Clinical stage & & & \\
$\quad$ I/II/III/IV & $84 / 202 / 150 / 77$ & & \\
\hline
\end{tabular}

turing agarose gels. TaqMan probe-based qPCR was performed using a commercial kit (Applied Biosystems; Thermo Fisher Scientific, Inc.) according to the manufacturer's protocol.

2.4. $q R T-P C R$ Procedure. The $\mathrm{qRT}$-PCR procedure was performed using a miR-28-5p specific primer and ABI's TaqMan MicroRNA Reverse Transcription kit (Applied Biosystems; Thermo Fisher Scientific, Inc.). U6 was used as the internal control. The following primers were used: miR28-5p forward, 5'-GGT AAG TCA CGCGGT-3' and reverse, $5^{\prime}$-CAG TGC GTC TCG TGG AGT-3 ${ }^{\prime}$; U6 forward, $5^{\prime}$-CTC GCT TCG GCA GCA CA-3' and reverse, $5^{\prime}$-AAC GCT TCA CGA ATT TGC GT-3'. The reaction conditions included 1 cycle at $95^{\circ} \mathrm{C}$ for 5 minutes; 15 cycles at $95^{\circ} \mathrm{C}$ for 25 seconds, $64^{\circ} \mathrm{C}$ for 20 seconds, $72^{\circ} \mathrm{C}$ for 20 seconds; and a final 31 cycles at $93^{\circ} \mathrm{C}$ for 25 seconds, $64^{\circ} \mathrm{C}$ for 20 seconds, $72^{\circ} \mathrm{C}$ for 20 seconds. Amplicons were detected using capillary electrophoresis on an ABI 3130xl Genetic Analyzer (Applied Biosystems/Life Technologies, Grand Island, NY). miR-28$5 \mathrm{p}$ levels were quantified using the $2-\Delta \Delta \mathrm{Cq}$ method.

2.5. Analysis of miR-28-5p in Colon Cancer. The expression of miR-28-5p was firstly compared among gastrointestinal cancers using the data from the TCGA database, including COAD, ESCA, LIHC, READ, CHOL, and STAD. Then, the association of miR-28-5p with clinical features was analyzed using the data from TCGA, GEO database, and our center. Next, the prognostic value of miR-28-5p in patients with colon cancer was analyzed, and the jointed effect of the combination of miR-28-5p with clinical features was performed. Finally, the predictive value of miR-28-5p on clinical features was analyzed.

2.6. miR-28-5p Target Genes Prediction and Functional Analysis. To explore the role of miR-28-5p in the diseases 


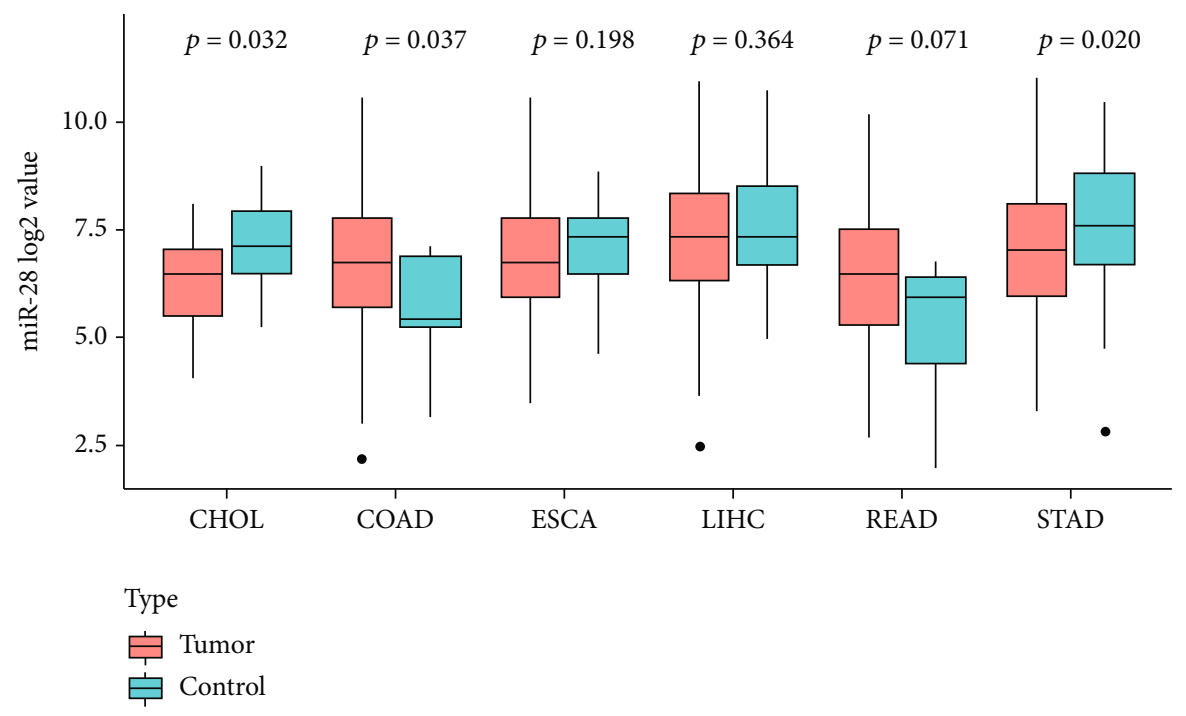

(a)

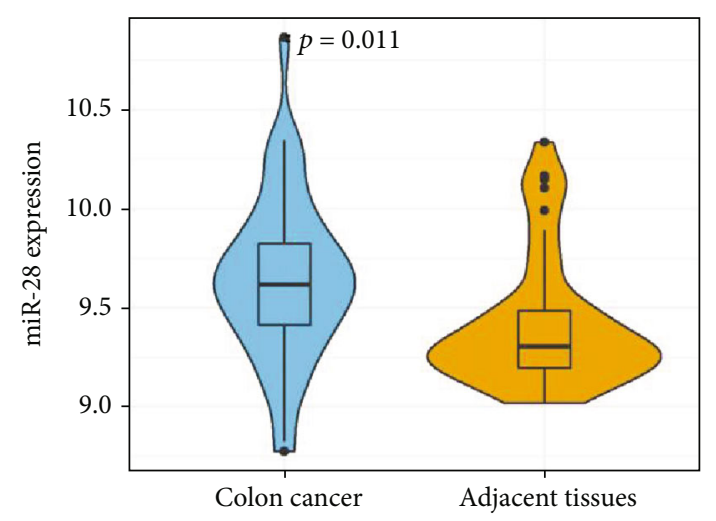

(b)

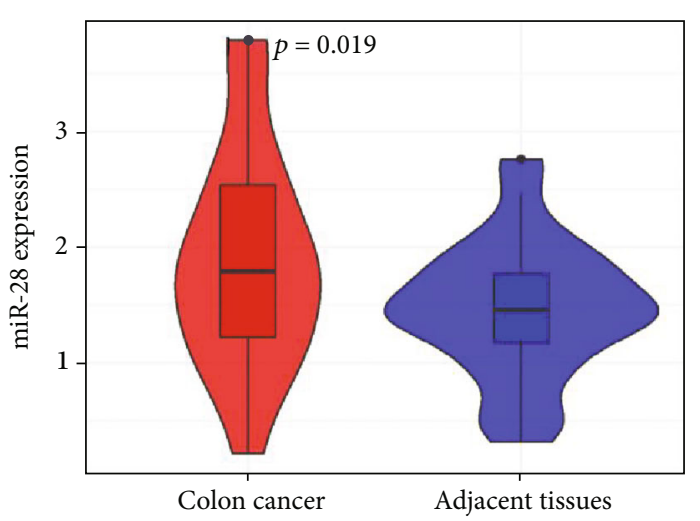

(c)

FIGURE 1: (a) Expression of miR-28-5p in gastrointestinal cancers. (b) Comparison of miR-28-5p in colon cancer and adjacent normal tissues from GSE49246. (c) Comparison of miR-28-5p in colon cancer and adjacent normal tissues from our center.

and the possible mechanism, we examined the function of miR-28-5p target genes and the pathway they involve. The target genes of miR-28-5p were predicted using the mirDIP database (http://ophid.utoronto.ca/mirDIP/), which integrated 15 miRNA prediction databases [16]. Then, the function of target genes was analyzed using Gene Ontology (GO) analysis, including molecular function (MF), biological process (BP) and cellular component (CC), and the Kyoto Encyclopedia of Genes and Genomes (KEGG) pathway was also analyzed using clusterProfiler package [17] running in $R$ language (version 3.5.2).

2.7. Statistical Analysis. Data are presented as the mean \pm standard deviation. The $\chi 2$ was used to compare the differences of categorical variables and the Student's $t$ test was used for comparison of differences between two groups. Kaplan-Meier survival curves and the log-rank test were used to analyze the survival rate in patients with colon cancer. Multivariate Cox proportional hazards regression models were performed to explore the prognostic value of multiple variables in colon cancer patients. The predictive value of miR-28-5p in clinical features was using the receiver operating characteristic (ROC) curve. All statistical analyses were performed using the $R$ language (version 3.4.1). $p<0.05$ was considered to indicate a statistically significant difference.

\section{Results}

3.1. Expression of miR-28-5p in Gastrointestinal Cancers. Table 1 listed the baseline data in patients with colon cancer. All the histopathological types of colon cancer were colon adenocarcinoma in three datasets, and the stage of COAD was assessed based on the American Joint Committee on Cancer criteria, 8th Edition. By analyzing the expression value of miR-28-5p in gastrointestinal cancer using the TCGA dataset, we found that the expression of miR-28-5p was significantly increased in COAD compared with corresponding normal tissues $(p=0.037)$, but we failed to find significant differences in ESCA $(p=0.198)$, LIHC $(p=0.364)$, and READ $(p=0.071)$, while expression of miR-28-5p was decreased in CHOL and STAD compared with the corresponding normal tissues $(p=0.032)$. The data from GSE49246 and our clinical colon cancer tissues also revealed 
TABLE 2: Association of miR-28-5p with clinical parameters in colon cancer patients.

\begin{tabular}{|c|c|c|c|c|c|c|}
\hline & TCGA & $p$ value & GSE49246 & $p$ value & Our data & $p$ value \\
\hline \multicolumn{7}{|l|}{ Age } \\
\hline$>60$ years & $105.3 \pm 30.0$ & 0.004 & $9.6 \pm 0.5$ & 0.621 & $2.21 \pm 0.87$ & 0.048 \\
\hline$\leq 60$ years & $115.1 \pm 33.7$ & & $9.6 \pm 0.3$ & & $1.63 \pm 0.92$ & \\
\hline \multicolumn{7}{|l|}{ Gender } \\
\hline Male & $106.4 \pm 30.9$ & 0.170 & $9.6 \pm 0.3$ & 0.827 & $1.72 \pm 1.06$ & 0.138 \\
\hline Female & $110.6 \pm 32.0$ & & $9.7 \pm 0.5$ & & $2.09 \pm 0.72$ & \\
\hline \multicolumn{7}{|l|}{ Location } \\
\hline Right site & $104.44 \pm 31.11$ & 0.048 & & & $1.77 \pm 0.87$ & 0.213 \\
\hline Left site & $110.08 \pm 32.84$ & & & & $2.07 \pm 1.36$ & \\
\hline \multicolumn{7}{|l|}{$\mathrm{T}$ stage } \\
\hline $\mathrm{T} 1+\mathrm{T} 2$ & $108.8 \pm 33.7$ & 0.89 & & & $1.66 \pm 0.80$ & 0.199 \\
\hline $\mathrm{T} 3+\mathrm{T} 4$ & $108.3 \pm 30.9$ & & & & $2.14 \pm 0.46$ & \\
\hline \multicolumn{7}{|l|}{$\mathrm{N}$ stage } \\
\hline N0 & $107.0 \pm 30.9$ & 0.276 & & & $1.61 \pm 0.73$ & 0.152 \\
\hline $\mathrm{N} 1+\mathrm{N} 2$ & $110.4 \pm 32.2$ & & & & $2.06 \pm 1.05$ & \\
\hline \multicolumn{7}{|l|}{ M stage } \\
\hline M0 & $106.4 \pm 31.6$ & 0.026 & & & $1.47 \pm 0.56$ & 0.004 \\
\hline $\mathrm{M} 1+\mathrm{MX}$ & $114.1 \pm 30.3$ & & & & $2.16 \pm 1.04$ & \\
\hline \multicolumn{7}{|l|}{ Clinical stage } \\
\hline $\mathrm{I}+\mathrm{II}$ & $110.8 \pm 31.8$ & 0.161 & & & $1.55 \pm 0.68$ & 0.005 \\
\hline $\mathrm{III}+\mathrm{IV}$ & $106.4 \pm 31.6$ & & & & $2.83 \pm 0.92$ & \\
\hline
\end{tabular}

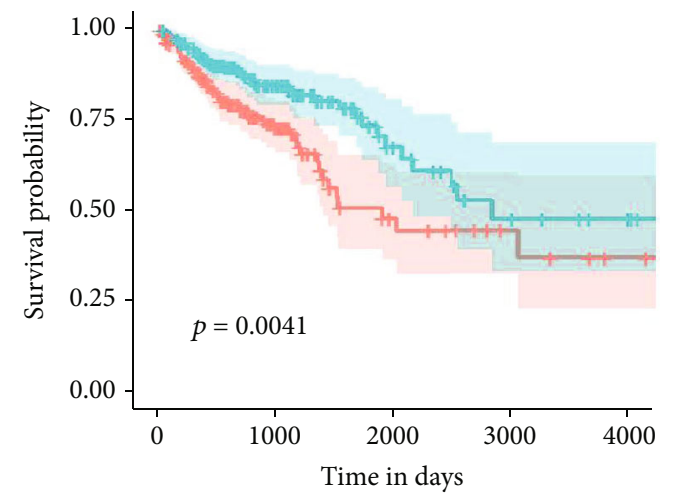

miR-28 expression

$$
\begin{aligned}
& + \text { High } \\
& + \text { Low }
\end{aligned}
$$

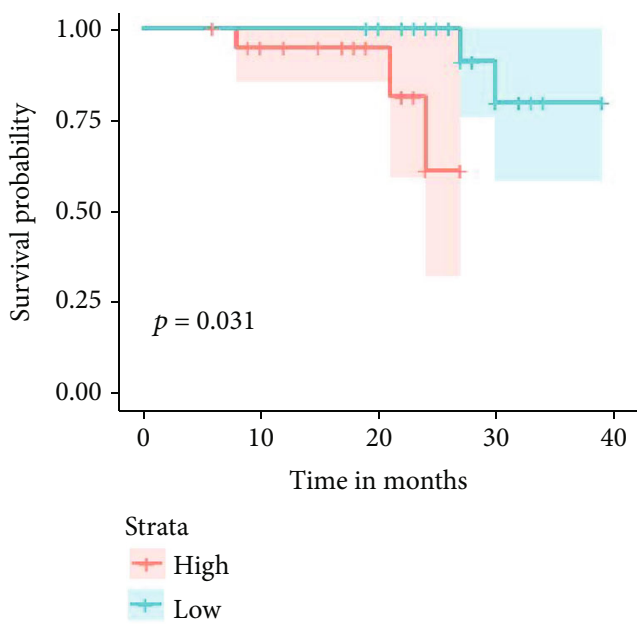

(b)

FIGURE 2: (a) Survival analysis of miR-28-5p in colon cancer patients using TCGA data. (b) Survival analysis of miR-28-5p in colon cancer patients using clinical tissue data.

that miR-28-5p was increased in colon cancer tissues compared with adjacent normal tissues (GSE49246: $p=0.011$; our data: $p=0.019)$. Figures $1(b)$ and $1(c)$. These results suggesting that miR-28-5p was increased in colon cancer.

3.2. The Association of miR-28-5p with Clinical Features. As shown in Table 2, the expression of miR-28-5p in the TCGA COAD dataset was significant difference regarding the patients' age and $M$ stage $(p<0.05)$, and the expression of miR-28-5p in elder patients and patients at M1 stage was higher than in young patients and patients at M0 stage. However, a not significant difference was found regarding the $\mathrm{T}$ stage, $\mathrm{N}$ stage, and clinical stage $(p>0.05)$. The data of GSE49246 showed that the expression of miR-28-5p has no obvious difference in the patient's gender and age $(p>0.05)$. The similar results 

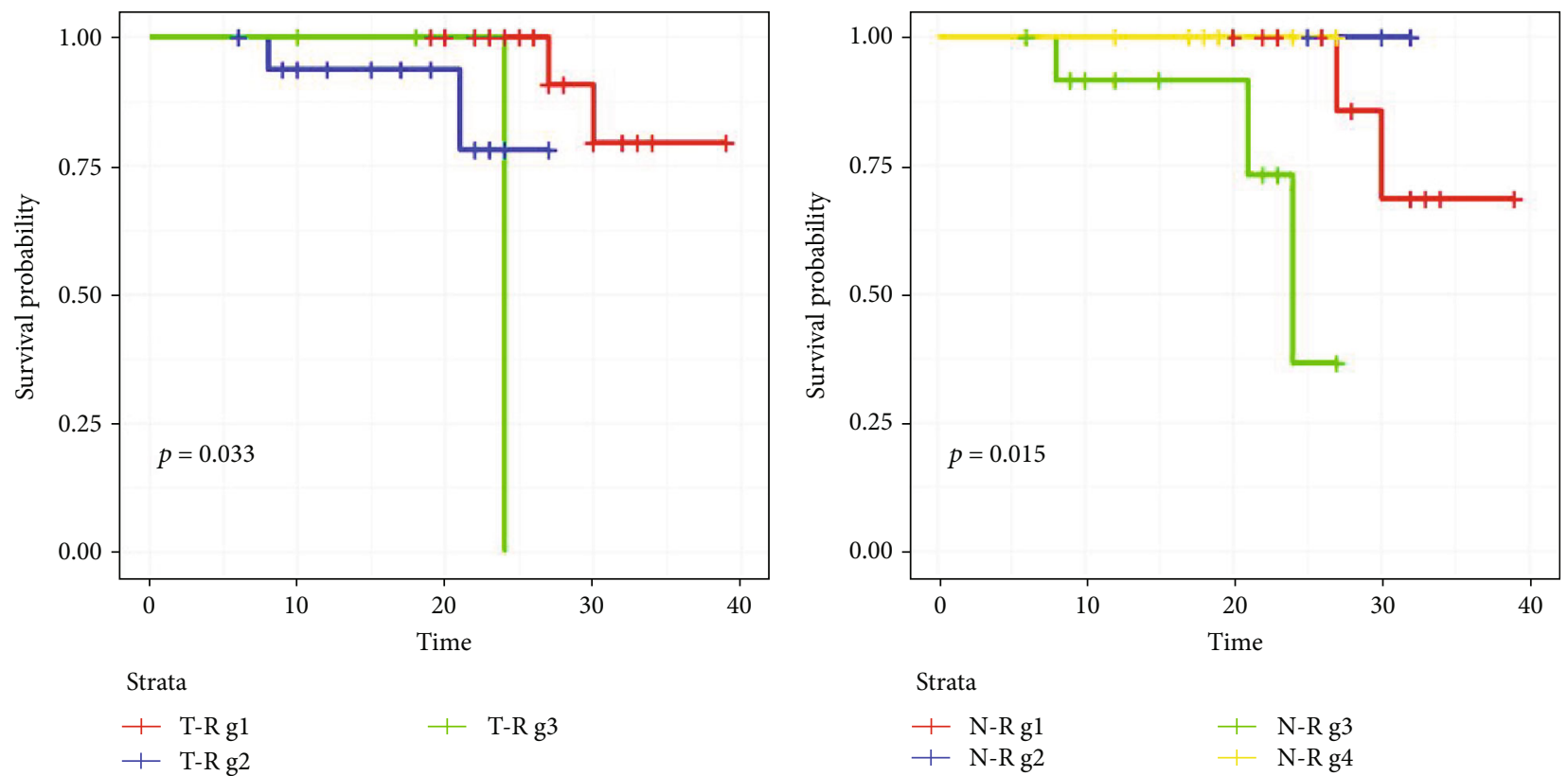

(a)
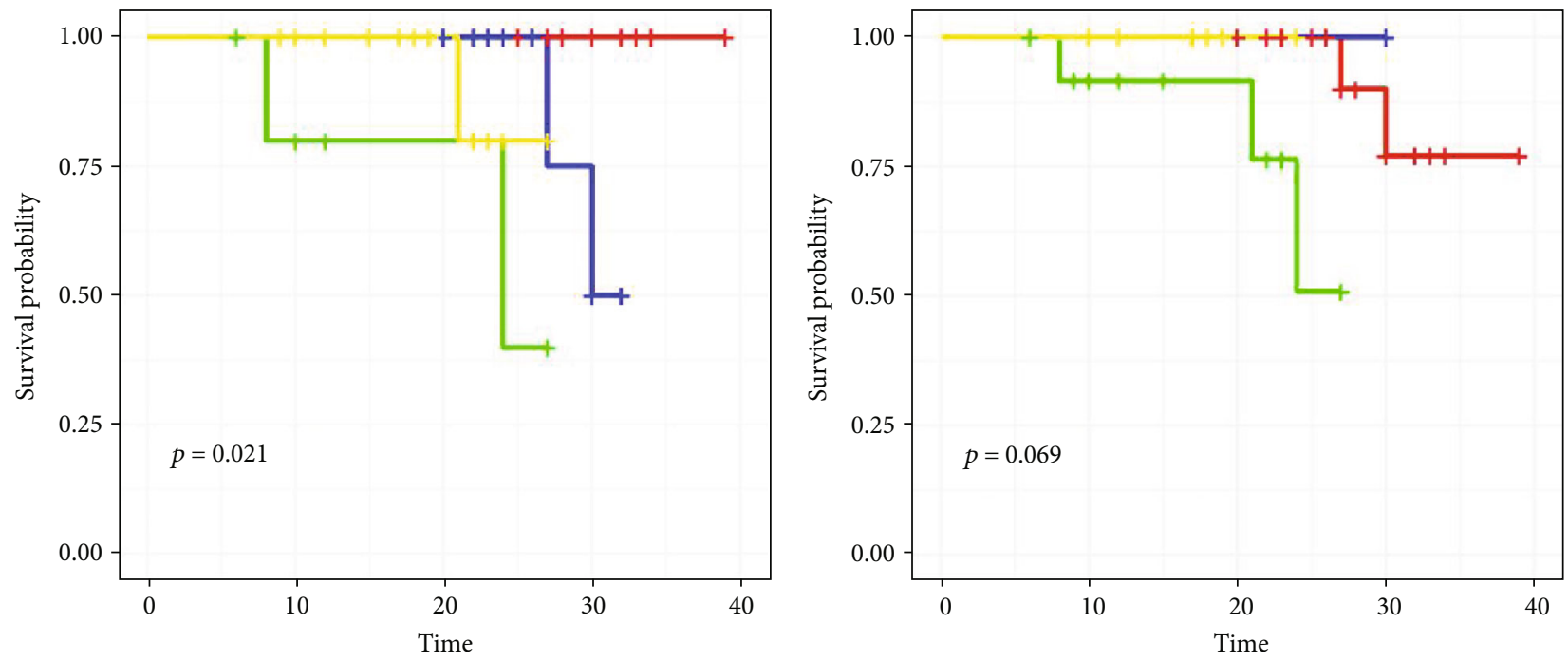

Strata

$\begin{array}{ll}+ \text { M-Rg1 } & + \text { M-R g3 } \\ + \text { M-Rg2 } & + \text { M-R g4 }\end{array}$

(c)

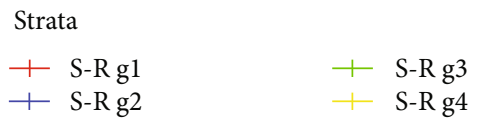

(d)

Figure 3: Kaplan-Meier survival curves for the combination of miR-28-5p with clinical features. (a) Combination of miR-28 with $\mathrm{T}$ stage. T-R g1: miR28-high+T stage-low; T-R g2: miR28-high+T stage-high; T-R g3: miR28-low+T stage-high; T-R g4: miR28-low+T stage-low. (b) Combination of miR-28 with $\mathrm{N}$ stage. N-R g1: miR28-high+N stage-low; N-R g2: miR28-high+N stage-high; N-R g3: miR28-low $+\mathrm{N}$ stage-high; N-R g4: miR28-low $+\mathrm{N}$ stage-low. (c) Combination of miR-28 with M stage. M-R g1: miR28-high+M stage-low; M-R g2: miR28-high+M stage-high; M-R g3: miR28-low+M stage-high; M-R g4: miR28-low+M stage-low. (d) Combination of miR-28 with clinical stage. S-R g1: miR28-high+clinical stage-low; S-R g2: miR28-high+clinical stage-high; S-R g3: miR28-low+clinical stage-high; S-R g4: miR28-low+clinical stage-low.

were observed in our data, and our results also found that the expression of miR-28-5p was remarkably increased in cancer at advance clinical stage (III+IV) compared with early clinical stage (I+II), with the $p$ value as 0.005 , indicating that miR-28-5p was closely related to the distant metastasis of colon cancer.
3.3. Prognostic Value of miR-28-5p in Patients with Colon Cancer. The results of the TCGA dataset showed that high expression of miR-28-5p indicated a shorter survival in patients with colon cancer; with the log-rank $p$ values as 0.004 , this result was confirmed by our data, and the logrank values were 0.031 . The multivariant Cox regression 
TABle 3: Multivariate Cox analysis for the combination of miR-2858 and clinical features in colon cancer patients.

\begin{tabular}{lccc}
\hline & HR & $95 \%$ CI & $p$ value \\
\hline miR-28/high+T/low & - & - & - \\
miR-28/high+T/high & 2.25 & $0.69-7.31$ & 0.178 \\
miR-28/low+T/low & 0.35 & $0.03-3.38$ & 0.366 \\
miR-28/low+T/high & 4.11 & $1.28-13.17$ & 0.017 \\
miR-28/high+N/low & - & - & - \\
miR-28/high+N/high & 1.52 & $0.81-2.86$ & 0.187 \\
miR-28/low+N/low & 0.72 & $0.36-1.42$ & 0.348 \\
miR-28/low+N/high & 3.27 & $1.85-5.78$ & $<0.001$ \\
miR-28/high+M/low & - & - & - \\
miR-28/high+M/high & 1.73 & $0.87-3.47$ & 0.116 \\
miR-28/low+M/low & 0.72 & $0.41-1.26$ & 0.252 \\
miR-28/low+M/high & 3.36 & $1.96-5.76$ & $<0.001$ \\
miR-28/high+stage/low & - & - & - \\
miR-28/high+stage/high & 2.02 & $1.01-4.05$ & 0.045 \\
miR-28/low+stage/low & 0.97 & $0.46-2.02$ & 0.94 \\
miR-28/low+stage/high & 4.77 & $2.55-8.92$ & $<0.001$ \\
\hline
\end{tabular}

analysis for the TCGA and our data further revealed that miR-28-5p was an independent factor for the prognosis in patients with colon cancer (TCGA dataset: $p=0.014$; our dataset: $p=0.049$ ), suggesting that miR-28-5p was a good prognostic indicator for the overall survival in patients with colon cancer. See Figure 2.

3.4. Joints Effect of Combination of miR-28-5p with Clinical Features. In order to identify more indicators that predicting the prognosis of colon cancer, we combined the expression of miR-28-5p with TNM stage and clinical stage using the data from TCGA and found that different combinations of miR28-5p with TNM stage and clinical stage were associated with the survival in patients with colon cancer (Figure 3). The multivariate Cox regression also revealed that these combinations could be acted as independent prognostic indicators in patients with colon cancer $(p<0.05)$ (Table 3$)$.

3.5. Predictive Value of miR-28-5p on Clinical Features. Due to the small sample size of our data, we used the data from TCGA to examine the relationship between the miR-28-5p and clinical features. We divided the clinical features into binary variable and tested the predictive value of miR-28$5 p$ using ROC analysis. As shown in Figures 4(a)-4(d), the predictive value of the miR-28-5p on these clinical features was moderated, with $\mathrm{T}$ stage $(\mathrm{AUC}=0.515), \mathrm{N}$ stage (AUC $=0.523), \mathrm{M}$ stage $(\mathrm{AUC}=0.572)$, and clinical stage $(\mathrm{AUC}=0.539)$, respectively.

3.6. Functional Analysis of miR-28-5p Target Genes. The mirDIP database identified 711 target genes of miR-28-5p (Supplementary Materials (available here)); we selected the first 400 target genes for the functional analysis. The GO analysis showed that the most enrichment of BP is the modulation of chemical synaptic transmission, and the CC is presynapse;
MF is protein serine/threonine kinase activity. The most enrichment of the KEGG pathway is the PI3K-Akt signaling pathway (Figure 5).

\section{Discussion}

There are several advantages of using miRNAs as prognostic biomarkers in cancers compared with mRNA and protein. First, the number of candidate miRNAs is much smaller than the protein-coding mRNA. Second, miRNAs are highly stable, especially in formalin-fixed, paraffin-embedded tissues compare with mRNAs. Third, qRT-PCR method is high sensitivity, cost-efficient in the detection of miRNAs. The limitations including that the samples must pass quality controls to avoid high degradation of nucleic acids or contamination of other miRNAs released by other cells or tissues [18].

miR-28-5p is an intragenic miRNA, which has been reported to be downregulated expressed in several tumor types, such as hepatocellular carcinoma [19] and renal cell carcinoma [20], but also upregulated in some other cancers, such as ovarian, esophageal, and cervical cancer [9, 21, 22]. With regard to the colon cancer, the expression of miR-28$5 \mathrm{p}$ was inconsistent in previous studies $[14,15]$. The reason of the inconsistent results can be attributed to the different sample or the detected methods, or different sample types, such as tissue specimen or serum sample. In this study, we determined the expression of miR-28-5p in colon cancer tissues using data from TCGA dataset, GEO dataset, respectively, which is using RNA sequencing or microarray technique to test the expression of miR-28-5p, and we also determined the expression of miR-28-5p in tumor tissues using the qRT-PCR method and performed functional analysis for the target genes of miR-28-5p, which provided a more reliable result compared with previous studies.

Previous studies have indicated that miR-28-5p regulated cell proliferation, migration, invasion, and in CRC $[23,24]$. In this study, by analyzing the data from TCGA, GEO dataset, and our clinical specimens, we found that the expression of miR-28-5p was increased in colon cancer tissues compared with corresponding normal tissues, suggesting that miR-28$5 \mathrm{p}$ may act as an oncogene during the process of colon carcinogenesis. This result is contrary to the Tsiakanikas et al. [14] report, who analyzed the expression of miR-28-5p in 182 CRC and 86 paired noncancerous colorectal mucosae. In addition, although no obvious difference among different $\mathrm{T}$ stage, $\mathrm{N}$ stage, we found that miR-28-5p was significantly increased in colon cancer in the M1 stage compared with the M0 stage, indicating that expression of miR-28-5p was associated with the progression of colon cancer.

Patient at advance TNM stage or clinical stage is likely to have a poor prognosis. Therefore, finding biomarkers that can detect colon cancer before advance TNM stage or clinical stage could increase the chances of early intervention and improve patient's survival. Currently, many miRNAs have been shown to be useful biomarkers for the early detection of CRC [25-27]. In a previous report [13], miR-28-5p in primary colon cancer tissues was showed to downregulate compared with the tissues of liver metastases. In a recent study, Wang et al. [28] reported that serum miR-28-5p expressions 


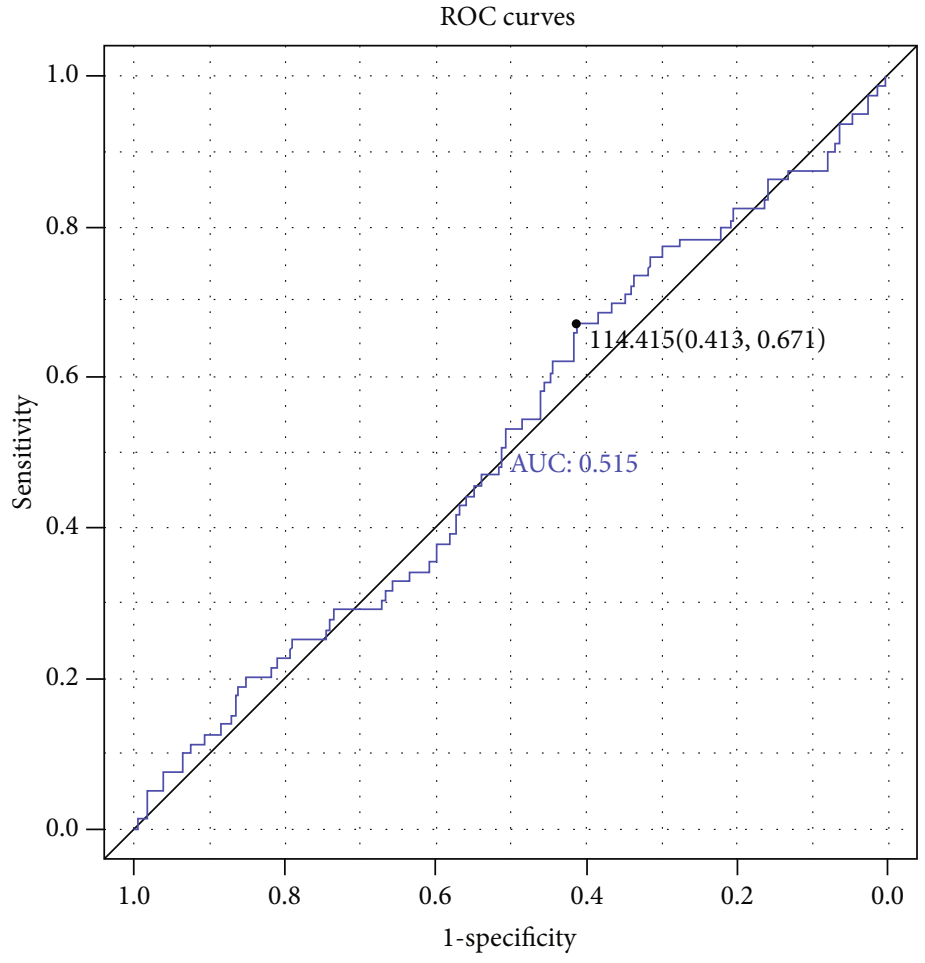

— T Stage

(a)

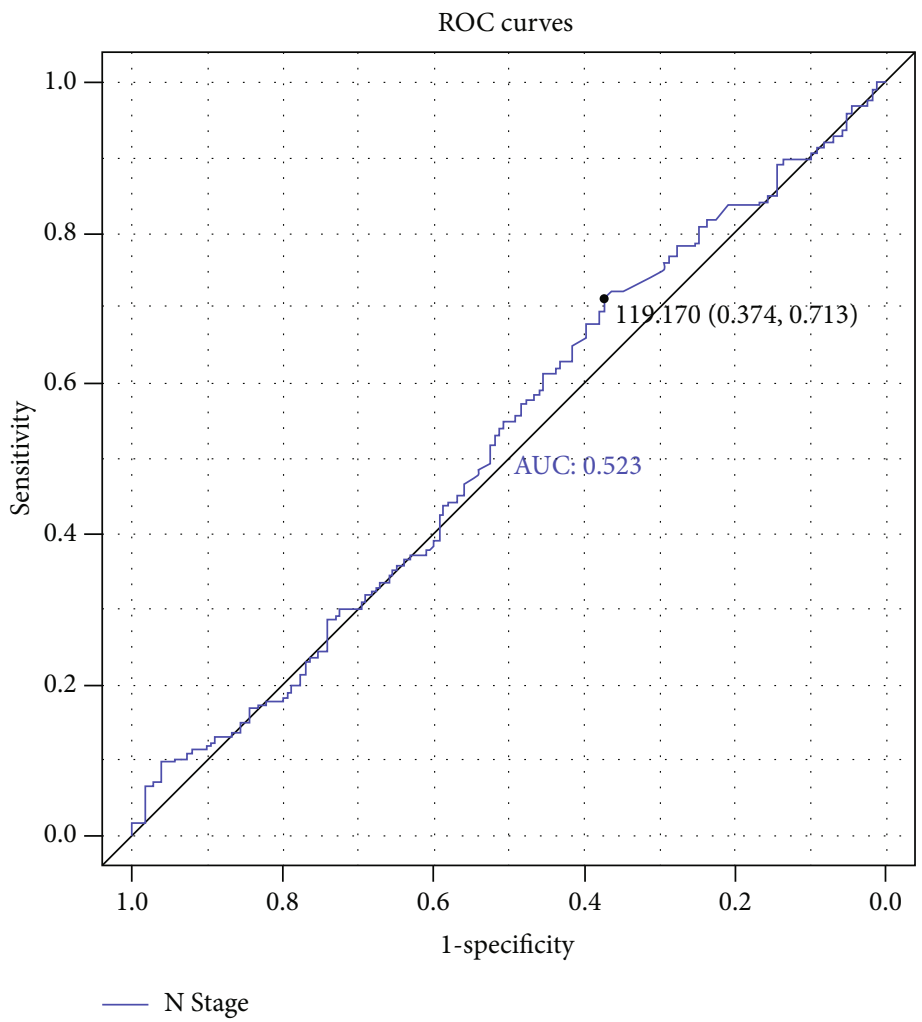

(b)

Figure 4: Continued. 


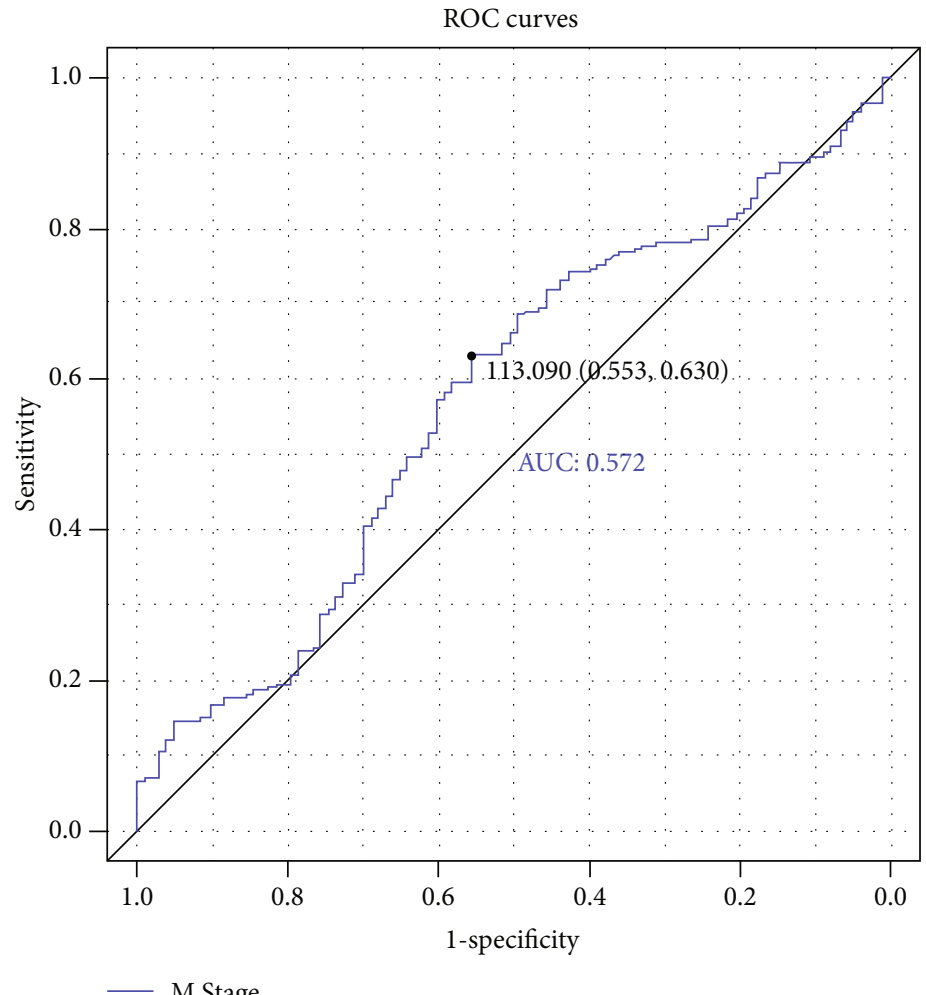

(c)

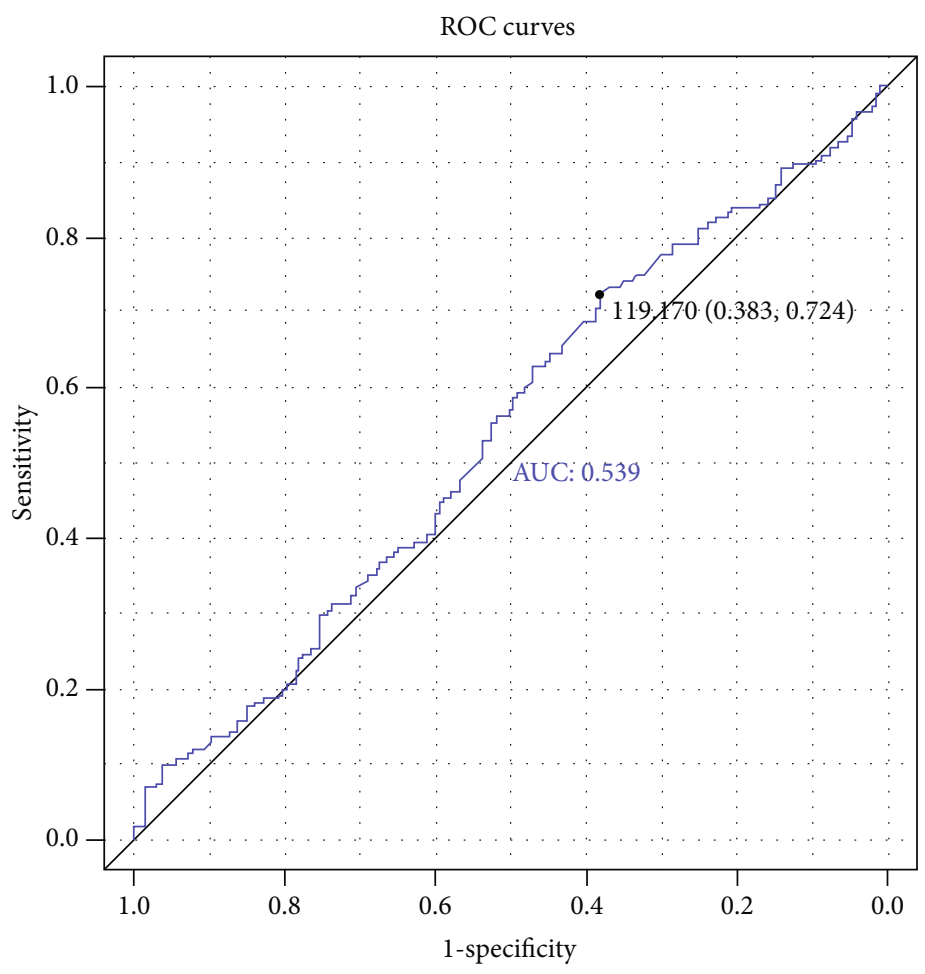

__ Clinical Stage

(d)

FIgUre 4: Predictive value of miR-28-5p on clinical features. (a) T stage. (b) N stage. (c) M stage. (d) Clinical stage. 


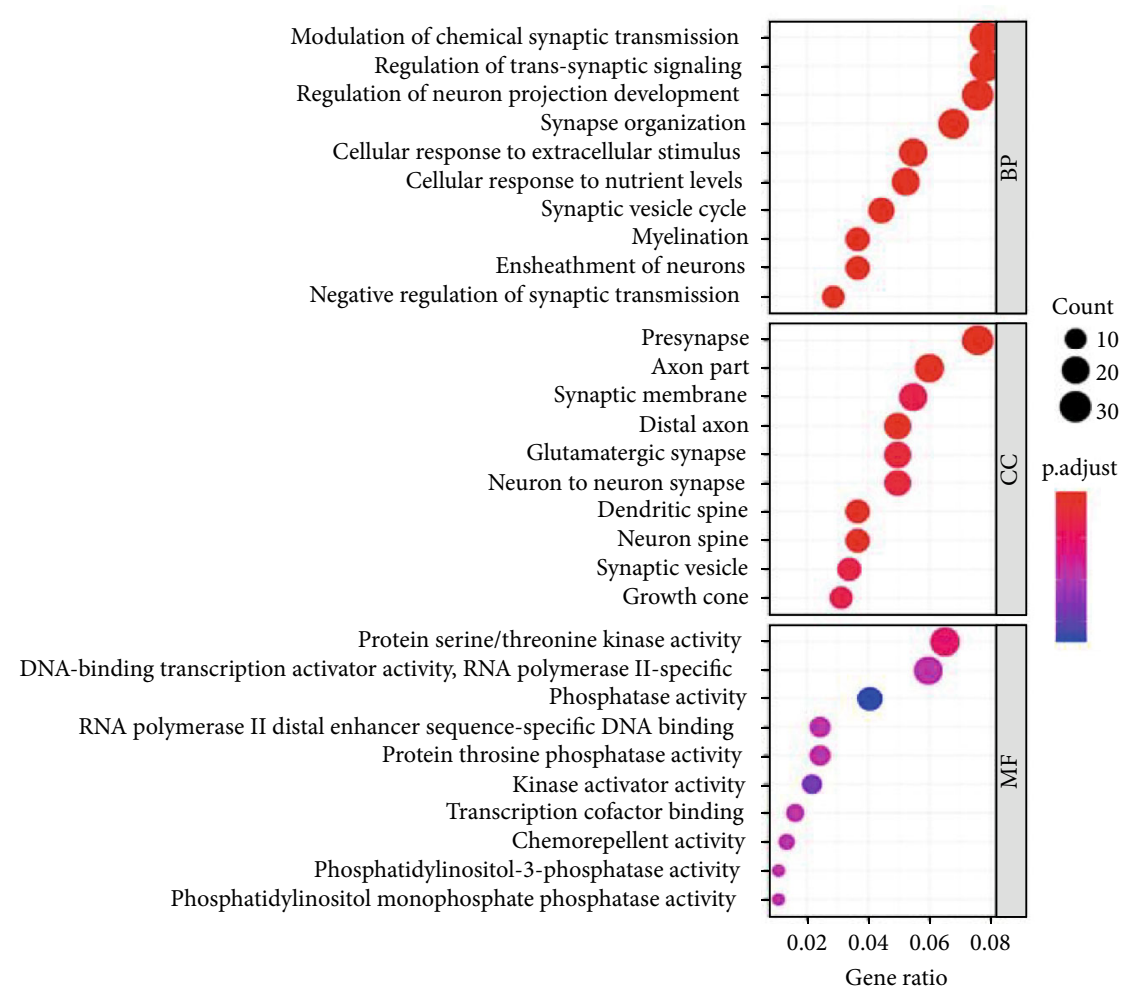

(a)

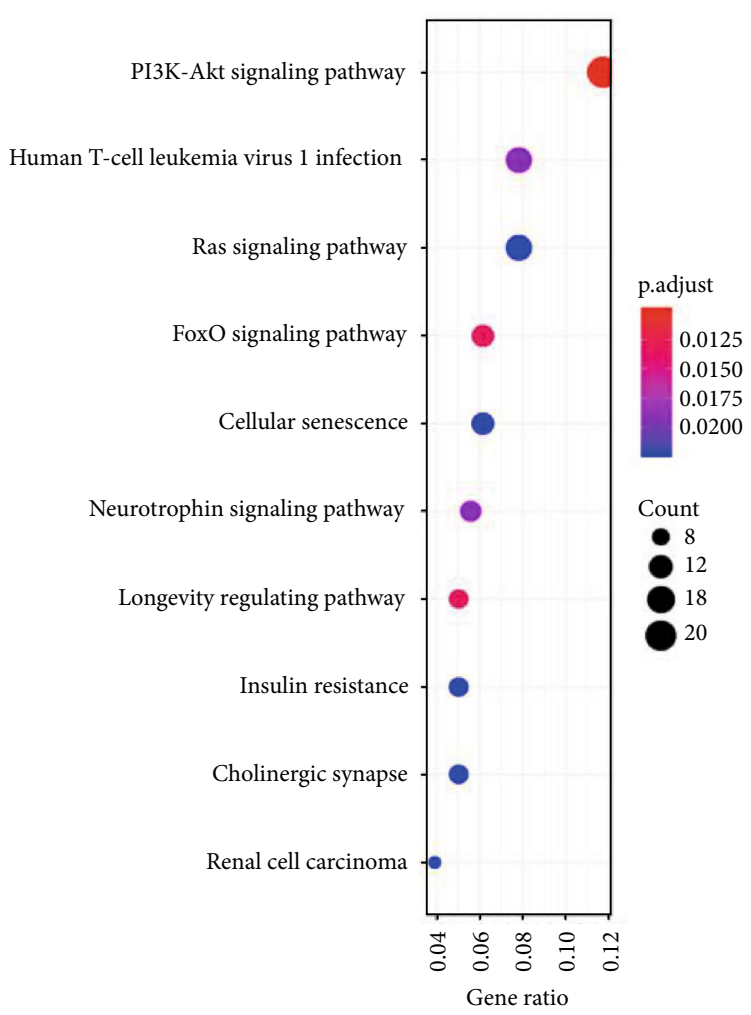

(b)

FIGURE 5: Functional analyses for the target genes of miR-28-5p. (a) Gene Ontology analysis for the target genes. (b) KEGG analysis for the target genes. 
were correlated with the TNM stage and liver metastasis. In this study, both our data and the TCGA data showed that miR-28-5p was associated with distant metastasis of colon cancer, although no association was found in lymph node metastasis, suggesting that high expression of miR-28-5p indicating a high chance of tumor distant metastasis of colon cancer.

Molecular biomarkers serve an important role in the therapeutic decision-making process, as they can be indicators for the patients to receive individual chemotherapeutic interventions. Although miR-28-5p has been reported to be aberrantly expressed in various human cancer $[8,9,11]$. However, limited knowledge is available about the association between miR-28-5p expression and the survival in patients with colon cancer. In the present study, we analyzed the prognostic value of miR-28-5p for patients with colon cancer and found that colon cancer patients with high expression of miR-28-5p were associated with a poor survival. Further analysis using multivariate Cox analysis also indicated that miR-28-5p was an independent indicator for the prognosis of colon cancer patients. These results were in agreement with Tsiakanikas et al. [14] report, which showed that high expression of miR-28-5p indicated poor disease-free survival and overall survival of CRC patients.

Since the above results showed that miR-28-5p was associated with the metastasis of colon cancer, we further examined the predictive value of miR-28-5p in the clinical features using ROC analysis. However, the results show the predictive value was moderate, including TNM stage and clinical stage, suggesting that we could not use miR-28-5p to clearly divide the patients into early or advanced TNM stage and clinical stage. Likewise, we also explored the combinations of miR-28-5p with the clinical features; by combining the miR-28-5p expression with TNM stage and clinical stage, we found that these combinations could clearly divide the patients into four groups and could be used as independent predictors to the survival in patients with colon cancers, suggesting that these combinations could significantly increase the prognostic value for patients with colon cancers.

Regarding the mechanism of miR-28-5p in the regulation of cancer cells, previous studies observed that miR-28-5p inhibited CAMTA2 expression and regulates colon cancer progression by suppressing $\mathrm{Wnt} / \beta$-catenin signaling [29]. As a direct target gene of miR-28-5p, SSRP1 promotes CRC progression and is negatively regulated by miR-28-5p [24]. miR-28-5p also reduced CRC cell proliferation, migration, and invasion in vitro by inhibiting CCND1 expression [30]. In the present study, we identified the potential target genes of miR-28-5p and revealed the function of these target genes through bioinformatics analysis; these results were helpful to uncover the possible mechanism of miR-28-5p in diseases.

Compared with Tsiakanikas et al. [14] study, our study only focus the colon cancer, and the results were validated by other datasets, which provided a more reliable results than previous studies. However, some limitations need to be noted. First, the sample size of our clinical colon cancer tissues was small; a larger number of samples was necessary to verify these results. Second, colon cancer is characteristic by microsatellite instability and chromosome instability, but our study did not analyze the impact of these factors. Third, some important gene mutation, such p53, K-ras mutation frequently occurs in the colon cancer, but we did not analyze the association between miR-28-5p and these genes. Fourth, although we conducted a functional analysis for the target genes of miR-28-5p using bioinformatics methods, the biological function of miR-28-5p needs to explore by using in vivo and in vitro experiments. Therefore, further study is needed by taking these factors into account in order to confirm the prognostic and early predict value of miR-28-5p in colon cancer.

\section{Conclusion}

The present study demonstrates that the expression miR-28$5 \mathrm{p}$ is increased in colon cancer and associated with the distant metastasis of cancer. High expression of miR-28-5p indicates a poor prognosis of colon cancer patients, which might serve as an independent indicator for the prognosis.

\section{Abbreviations \\ CRC: $\quad$ Colorectal cancer \\ TCGA: The Cancer Genome Atlas \\ GEO: $\quad$ Gene Expression Omnibus \\ OS: Overall survival \\ ROC: Receiver operating characteristic \\ CAMTA2: Calmodulin binding transcription activator 2 \\ CCND1: Cyclin D1 \\ qRT-PCR: Quantitative real-time polymerase chain reaction.}

\section{Data Availability}

Answer: Yes. Comment: The data used to support the findings of this study are included within the article. (Table 1).

\section{Conflicts of Interest}

The authors declare no competing financial interests.

\section{Authors' Contributions}

Study concept and design was presented by LJL and HBL. Collection and assembly of data was contributed by XMZ and LKZ. The experiment was performed by XMZ and TYL. Data analysis and interpretation was contributed HBL, TYP, and XMZ. All authors have written and reviewed the manuscript. Ji-lin Li and Ke-zhi Li contributed equally to this work.

\section{Acknowledgments}

This work was supported by the Guangxi Health Research Project (No. Z20180626; Z20180627; Z20180613).

\section{Supplementary Materials}

The mirDIP database identified the target genes of miR-285p. (Supplementary Materials) 


\section{References}

[1] F. Bray, J. Ferlay, I. Soerjomataram, R. L. Siegel, L. A. Torre, and A. Jemal, "Global cancer statistics 2018: GLOBOCAN estimates of incidence and mortality worldwide for 36 cancers in 185 countries," CA: a Cancer Journal for Clinicians, vol. 68, no. 6, pp. 394-424, 2018.

[2] L. Duan, W. Yang, X. Wang et al., "Advances in prognostic markers for colorectal cancer," Expert Review of Molecular Diagnostics, vol. 19, no. 4, pp. 313-324, 2019.

[3] A. Jemal, E. M. Ward, C. J. Johnson et al., "Annual report to the nation on the status of cancer, 1975-2014, featuring survival," Journal of the National Cancer Institute, vol. 109, no. 9, 2017.

[4] Y. Choi, H. F. Sateia, K. S. Peairs, and R. W. Stewart, "Screening for colorectal cancer," Seminars in Oncology, vol. 44, no. 1, pp. 34-44, 2017.

[5] H. Xie, J. H. Guo, W. M. An et al., "Diagnostic value evaluation of trefoil factors family 3 for the early detection of colorectal cancer," World Journal of Gastroenterology, vol. 23, no. 12, pp. 2159-2167, 2017.

[6] K. K. W. To, C. W. S. Tong, M. Wu, and W. C. S. Cho, "MicroRNAs in the prognosis and therapy of colorectal cancer: from bench to bedside," World Journal of Gastroenterology, vol. 24, no. 27, pp. 2949-2973, 2018.

[7] P. Krawczyk, T. Powrózek, T. Olesiński et al., "Evaluation of miR-506 and miR-4316 expression in early and non-invasive diagnosis of colorectal cancer," International Journal of Colorectal Disease, vol. 32, no. 7, pp. 1057-1060, 2017.

[8] S. M. Rapti, C. K. Kontos, S. Christodoulou, I. N. Papadopoulos, and A. Scorilas, "miR-34a overexpression predicts poor prognostic outcome in colorectal adenocarcinoma, independently of clinicopathological factors with established prognostic value," Clinical Biochemistry, vol. 50, no. 16-17, pp. 918924, 2017.

[9] J. Xu, N. Jiang, H. Shi, S. Zhao, S. Yao, and H. Shen, "miR-28$5 p$ promotes the development and progression of ovarian cancer through inhibition of N4BP1," International Journal of Oncology, vol. 50, no. 4, pp. 1383-1391, 2017.

[10] M. Rizzo, G. Berti, F. Russo, M. Evangelista, M. Pellegrini, and G. Rainaldi, "The miRNA pull out assay as a method to validate the miR-28-5p targets identified in other tumor contexts in prostate cancer," International Journal of Genomics, vol. 2017, Article ID 5214806, 7 pages, 2017.

[11] S. L. Zhou, Z. Q. Hu, Z. J. Zhou et al., "miR-28-5p-IL-34-macrophage feedback loop modulates hepatocellular carcinoma metastasis," Hepatology, vol. 63, no. 5, pp. 1560-1575, 2016.

[12] J. M. Sayagués, L. A. Corchete, M. L. Gutiérrez et al., "Genomic characterization of liver metastases from colorectal cancer patients," Oncotarget, vol. 7, no. 45, pp. 72908-72922, 2016.

[13] P. Vychytilova-Faltejskova, M. Pesta, L. Radova et al., "Genome-wide microRNA expression profiling in primary tumors and matched liver metastasis of patients with colorectal Cancer," Cancer Genomics \& Proteomics, vol. 13, no. 4, pp. 311-316, 2016.

[14] P. Tsiakanikas, C. K. Kontos, D. Kerimis, I. N. Papadopoulos, and A. Scorilas, "High microRNA-28-5p expression in colorectal adenocarcinoma predicts short-term relapse of nodenegative patients and poor overall survival of patients with non-metastatic disease," Clinical Chemistry and Laboratory Medicine, vol. 56, no. 6, pp. 990-1000, 2018.
[15] J. X. Zhang, W. Song, Z. H. Chen et al., "Prognostic and predictive value of a microRNA signature in stage II colon cancer: a microRNA expression analysis," The Lancet Oncology, vol. 14, no. 13, pp. 1295-1306, 2013.

[16] T. Tokar, C. Pastrello, A. E. M. Rossos et al., "mirDIP 4.1-integrative database of human microRNA target predictions," Nucleic Acids Research, vol. 46, no. D1, pp. D360-D370, 2018.

[17] G. Yu, L. G. Wang, Y. Han, and Q. Y. He, "clusterProfiler: an R package for comparing biological themes among gene clusters," OMICS, vol. 16, no. 5, pp. 284-287, 2012.

[18] J. L. García-Giménez, M. Seco-Cervera, T. O. Tollefsbol et al., "Epigenetic biomarkers: current strategies and future challenges for their use in the clinical laboratory," Critical Reviews in Clinical Laboratory Sciences, vol. 54, no. 7-8, pp. 529-550, 2017.

[19] X. Shi and F. Teng, "Down-regulated miR-28-5p in human hepatocellular carcinoma correlated with tumor proliferation and migration by targeting insulin-like growth factor-1 (IGF1)," Molecular and Cellular Biochemistry, vol. 408, no. 1-2, pp. 283-293, 2015.

[20] C. Wang, C. Wu, Q. Yang et al., "miR-28-5p acts as a tumor suppressor in renal cell carcinoma for multiple antitumor effects by targeting RAP1B," Oncotarget, vol. 7, no. 45, pp. 73888-73902, 2016.

[21] Z. Huang, L. Zhang, D. Zhu et al., "A novel serum microRNA signature to screen esophageal squamous cell carcinoma," Cancer Medicine, vol. 6, no. 1, pp. 109-119, 2017.

[22] S. M. Wilting, P. J. F. Snijders, W. Verlaat et al., "Altered microRNA expression associated with chromosomal changes contributes to cervical carcinogenesis," Oncogene, vol. 32, no. 1, pp. 106-116, 2013.

[23] M. Cui, M. Chen, Z. Shen, R. Wang, X. Fang, and B. Song, "LncRNA-UCA1 modulates progression of colon cancer through regulating the miR-28-5p/HOXB3 axis," Journal of Cellular Biochemistry, vol. 120, no. 5, pp. 6926-6936, 2018.

[24] W. Wu, K. He, Q. Guo et al., "SSRP1 promotes colorectal cancer progression and is negatively regulated by miR-28-5p," Journal of Cellular and Molecular Medicine, vol. 23, no. 5, pp. 3118-3129, 2019.

[25] S. Guo, J. Zhang, B. Wang et al., "A 5-serum miRNA panel for the early detection of colorectal cancer," OncoTargets and Therapy, vol. 11, pp. 2603-2614, 2018.

[26] H. Feng, M. Xu, Y. Zhang, B. Han, J. Wang, and P. Sun, "Identification of differentially expressed microRNAs involved in the pathogenesis of colorectal cancer," Clinical Laboratory, vol. 64, 2018.

[27] D. Dayde, I. Tanaka, R. Jain, M. C. Tai, and A. Taguchi, "Predictive and prognostic molecular biomarkers for response to neoadjuvant chemoradiation in rectal cancer," International Journal of Molecular Sciences, vol. 18, no. 3, p. 573, 2017.

[28] L. Wang, Y. Zhao, M. Xu, F. Zhou, and J. Yan, "Serum miR1301-3p, miR-335-5p, miR-28-5p, and their target B7-H3 may serve as novel biomarkers for colorectal cancer," Journal of BUON, vol. 24, no. 3, pp. 1120-1127, 2019.

[29] X. F. Luan, L. Wang, and X. F. Gai, “The miR-28-5p-CAMTA2 axis regulates colon cancer progression via $\mathrm{Wnt} / \beta$-catenin signaling," Journal of Cellular Biochemistry, 2019.

[30] M. I. Almeida, M. S. Nicoloso, L. Zeng et al., "Strand-specific miR-28-5p and miR-28-3p have distinct effects in colorectal cancer cells," Gastroenterology, vol. 142, no. 4, pp. 886896.e9, 2012. 